\title{
Background thermal depolarization of electrons in storage rings
}

\author{
A. C. C. Guimarães, ${ }^{*}$ G. E. A. Matsas, ${ }^{\dagger}$ and D. A. T. Vanzella ${ }^{\ddagger}$ \\ Instituto de Física Teórica, Universidade Estadual Paulista, R. Pamplona 145, 01405-900-São Paulo, São Paulo, Brazil
}

(Received 3 March 1997; published 17 February 1998)

\begin{abstract}
We discuss the influence of the background thermal bath on the depolarization of electrons in high-energy storage rings, and on the photon emission associated with the spin flip. We focus, in particular, on electrons at CERN LEP. We show that in a certain interval of solid angles the photon emission is enhanced several orders of magnitude because of the presence of the thermal bath. Notwithstanding, the overall depolarization induced by the background thermal bath at LEP conditions is much smaller than the one induced by plain acceleration at zero temperature and can be neglected in practical situations. Eventually we discuss under what conditions the background thermal bath can enhance the overall depolarization by several orders of magnitude. [S0556-2821(98)03505-X]
\end{abstract}

PACS number(s): 41.60.- m, 12.20.Ds, 12.20.Fv

\section{INTRODUCTION}

Evidence of polarization in a single circulating beam was detected unambiguously in the early 1970s at Novosibirsk and Orsay [1]. Later, a polarization of $P \approx 76 \%$ was observed in the storage ring SPEAR at Stanford [2] and more recently a polarization of $P \approx 90 \%$ [3]. The first observation of transverse beam polarization at the CERN $e^{+} e^{-}$collider LEP was in 1990 [4], reaching further, $P \approx 57 \%$ [5]. Transverse and longitudinal polarization signals have been observed since then (see, e.g., [6] and references therein), and their utilization to test possible extensions to the standard model constitutes a source of excitement (see, e.g., [7]). In spite of the peculiarities of the different machines, theoretical calculations indicate that the maximum natural transverse polarization possible to be reached by ultrarelativistic electrons moving circularly in storage rings at zero temperature is $P \approx 92 \%$ [8-10]. The main reason why the polarization obtained is not complete is the high acceleration under which these electrons are subjected. However, there are other sources of depolarization which should be taken into account (see, e.g., [11]).

Here we discuss the contribution of the background thermal bath on the depolarization of high-energy electron beams at storage rings and on the photon emission associated with the spin flip. We focus on electrons at the LEP, but our conclusions will remain basically the same in most situations of interest. Theoretical results call attention to the fact that depending on the electron's velocity, the background thermal-bath contribution can be enhanced (or damped) by several orders of magnitude [12]. This result was obtained in a simplified context by modeling the electron's spin flip by the transition of a two-level scalar system [13] coupled to the background thermal bath. The influence of the velocity in the thermal depolarization rate can be understood by noticing that because of the Doppler effect the energy spectrum of the background photons is shifted in the electron's proper frame. Thus, depending on the electron's velocity, photons of the

\footnotetext{
*Email address: acandido@ift.unesp.br

†Email address: matsas@axp.ift.unesp.br

‡Email address: vanzella@axp.ift.unesp.br
}

background thermal bath can have their frequency shifted into or off the absorbable band, implying thus an enhancement or damping of the excitation rate. Although the twolevel model is a satisfactory approximation in many respects, this is incomplete in some other ones [14]. Here we aim to analyze the influence of the background thermal bath on true fast-moving spin- $1 / 2$ fermions.

The paper is organized as follows: In Sec. II, we calculate the angular distribution of emitted and absorbed photons, and radiated power induced by the spin flip. We show that in a certain interval of solid angles the photon emission induced by the spin flip is enhanced by several orders of magnitude because of the presence of the thermal bath. In Sec. III we exhibit the frequency distribution. Section IV is devoted to calculating the total emission rate and total radiated power induced by the spin flip. In Sec. V we use results of the previous sections to calculate the background thermal-bath influence on the depolarization of electrons at the LEP. We show that in spite of the results of Sec. II, the overall depolarization because of the background thermal bath at LEP conditions is much smaller than the one because of plain acceleration at zero temperature. Finally we discuss our results in Sec. VI. Natural units $\hbar=c=k=1$ will be adopted throughout the paper.

\section{PHOTON ANGLE DISTRIBUTION}

In order to calculate the angle distribution of emitted photons induced by the spin flip of a fast-moving electron, it is useful to define from the beginning spherical angular coordinates $(\theta, \phi)$ in an inertial frame at rest with the laboratory and with its origin instantaneously on the electron as follows: $\theta$ is the angle between the electron's three-velocity $\mathbf{v}$ and the three-momentum $\mathbf{k}$ of the emitted photon, while $\phi$ is the angle between the projection of $\mathbf{k}$ on the plane orthogonal to $\mathbf{v}$ and the electron's three-acceleration $\mathbf{a}$.

To calculate at the tree level the angular distribution of emitted and absorbed photons associated with the spin flip as well as the corresponding radiated power, rather than using the thermal Green function approach, we will introduce directly the proper thermal factor (Planck factor) in the vacuum probability distribution previously calculated by Jackson [10]: The photon emission rate per laboratory time $d \mathcal{P}_{\text {vac }}^{\mathrm{em}}$ per 
solid angle $d \Omega=\sin \phi d \theta d \phi$, and frequency $d \omega$ induced by the spin flip of an electron circulating in a storage ring at zero temperature is

$$
\begin{aligned}
\frac{d^{2} \mathcal{P}_{\mathrm{vac}}^{\mathrm{em}}\left(\theta_{0}\right)}{d \Omega d \omega}= & \frac{3 \sqrt{3}}{40 \pi^{3}} \frac{\nu^{3}\left(1+t^{2}\right)}{\tau_{0} \gamma^{2} \omega_{0}}\left\{\sin ^{2} \theta_{0} K_{1 / 3}^{2}(\eta)\right. \\
& +\frac{1}{2}\left(1+\cos ^{2} \theta_{0}\right)\left(1+t^{2}\right)\left[K_{1 / 3}^{2}(\eta)+K_{2 / 3}^{2}(\eta)\right] \\
& \left.+2 \cos \theta_{0} \sqrt{1+t^{2}} K_{1 / 3}(\eta) K_{2 / 3}(\eta)\right\}
\end{aligned}
$$

where $\gamma=1 / \sqrt{1-v^{2}}, t=\gamma \theta \sin \phi, \omega_{0}$ is the electron's orbital frequency,

$$
\tau_{0}=\left[\frac{5 \sqrt{3}}{8} \frac{e^{2} \gamma^{5}}{m^{2} \rho^{3}}\right]^{-1}
$$

is the typical time interval for the electron beam to reach polarization equilibrium $P_{0}$, i.e. $P(t)=P_{0}\left[1-\exp \left(-t / \tau_{0}\right)\right]$, $m$ is the electron's mass, $\rho$ is the bending radius of the storage ring, and $\eta=\nu\left(1+t^{2}\right)^{3 / 2} / 2$ with

$$
\nu \equiv \frac{2 \omega}{3 \gamma^{3} \omega_{0}}
$$

The variable $\theta_{0}$ is defined as being the angle between the initial spin direction $\mathbf{s}$ and the magnetic field $\mathbf{B}$. After any transition the spin state changes from $|\mathbf{s}\rangle$ to $|-\mathbf{s}\rangle$. Deexcitation processes are characterized by the fact that $0 \leqslant \theta_{0}$ $<\pi / 2$, while excitation processes are characterized by the fact that $\pi / 2<\theta_{0} \leqslant \pi$. We also recall that, at the tree level, each spin flip in the vacuum is associated with a photon emission [10]. For the LEP we have $\gamma \approx 10^{5}, \omega_{0} \approx 10^{5} \mathrm{~s}^{-1}$ and a background temperature of $\beta^{-1} \approx 4 \times 10^{13} \mathrm{~s}^{-1}(=300$ $\mathrm{K})$. Note that the transition energy gap, which corresponds to the typical photon energy responsible for the spin flip, can be naively estimated in the electron's proper frame (see Refs. [10] and [13]): $\Delta E=2 \mu\left|\mathbf{B}^{\prime}\right| \approx 0.6 \mathrm{eV}$, where $\mu=g e / 4 m$ is the magnetic moment, $g \approx 2.0023$ is the gyromagnetic factor, and $\mathbf{B}^{\prime}$ is the magnetic field in the instantaneously inertial rest frame of the electron. A more careful calculation [15] shows that $\Delta E$ is proportional to $g-2+2 / \gamma$ rather than $g$, which reduces by a factor of $10^{3}$ the energy gap for ultrarelativistic electrons. In any case $\Delta E \ll m \approx 0.5 \mathrm{MeV}$.

In the case the electron is moving in a background thermal bath characterized by a temperature $\beta^{-1}$, the emission rate can be expressed simply in terms of Eq. (2.1) by (see, e.g., [16] for an account of photon radiation in a heat bath)

$$
\frac{d^{2} \mathcal{P}^{\mathrm{em}}\left(\theta_{0}\right)}{d \Omega d \omega}=\frac{d^{2} \mathcal{P}_{\mathrm{vac}}^{\mathrm{em}}\left(\theta_{0}\right)}{d \Omega d \omega}+\frac{d^{2} \mathcal{P}_{\mathrm{ther}}^{\mathrm{em}}\left(\theta_{0}\right)}{d \Omega d \omega},
$$

$$
\begin{aligned}
\frac{d^{2} \mathcal{P}_{\text {ther }}^{\mathrm{em}}\left(\theta_{0}\right)}{d \Omega d \omega} & \equiv\left|\mathcal{A}_{\mathrm{vac}}^{\mathrm{em}}\left(\theta_{0}\right)\right|^{2} \omega^{2} n(\omega) \\
& =\frac{d^{2} \mathcal{P}_{\mathrm{vac}}^{\mathrm{em}}\left(\theta_{0}\right)}{d \Omega d \omega} n(\omega)
\end{aligned}
$$

accounts for stimulated emission, and $n(\omega)=1 /\left(e^{\omega \beta}-1\right)$. The emission amplitude can be written as

$$
\mathcal{A}_{\mathrm{vac}}^{\mathrm{em}}\left(\theta_{0}\right)=\left\langle-\mathbf{s}\left|\otimes\left\langle 1_{\mathbf{k}}\left|H_{I}\right| 0\right\rangle \otimes\right| \mathbf{s}\right\rangle,
$$

where $H_{I}$ is the relevant interaction Hamiltonian.

In the presence of a background thermal bath, the spin-flip process can be also related to the absorption of a photon. In order to calculate the absorption rate, we note that

$$
\frac{d^{2} \mathcal{P}^{\mathrm{abs}}\left(\theta_{0}\right)}{d \Omega d \omega} \equiv\left|\mathcal{A}_{\text {vac }}^{\mathrm{abs}}\left(\theta_{0}\right)\right|^{2} \omega^{2} n(\omega),
$$

where the absorption amplitude is written as

$$
\mathcal{A}_{\text {vac }}^{\text {abs }}\left(\theta_{0}\right)=\left\langle-\mathbf{s}\left|\otimes\left\langle 0\left|H_{I}\right| 1_{\mathbf{k}}\right\rangle \otimes\right| \mathbf{s}\right\rangle .
$$

By comparing Eq. (2.7) with Eq. (2.9), and recalling that if the angle between $\mathbf{s}$ and $\mathbf{B}$ is $\theta_{0}$ then the angle between $-\mathbf{S}$ and $\mathbf{B}$ is $\pi-\theta_{0}$, we obtain

$$
\mathcal{A}_{\mathrm{vac}}^{\mathrm{abs}}\left(\theta_{0}\right)=\overline{\mathcal{A}_{\mathrm{vac}}^{\mathrm{em}}\left(\pi-\theta_{0}\right)} \text {. }
$$

By using it in conjunction with Eqs. (2.5) and (2.8) we obtain that the absorption probability with spin excitation (deexcitation) must be equal to the stimulated emission probability with spin deexcitation (excitation):

$$
\frac{d^{2} \mathcal{P}^{\mathrm{abs}}\left(\theta_{0}\right)}{d \Omega d \omega}=\frac{d^{2} \mathcal{P}_{\mathrm{ther}}^{\mathrm{em}}\left(\pi-\theta_{0}\right)}{d \Omega d \omega} .
$$

The total spin-flip probability will be given by summing up Eqs. (2.4) and (2.10), and integrating the result over the frequency $\omega$ and solid angle $\Omega$ as shown in Sec. IV.

In order to obtain the angle distribution of emitted photons, we integrate Eq. (2.4) over frequencies $\omega$. In the $|t|$ $<50$ interval, we use the approximation $K_{a>0}(\eta \ll 1)$ $\approx \Gamma(a) 2^{a-1} / \eta^{a}$ since $n(\omega \beta \gg 1) \sim e^{-\omega \beta}$ implies that the integral has its main contribution for $0<\omega \beta<10$ and in this range $\eta \ll 1$. In the $|t|>9 \times 10^{2}$ interval, we use the approximation $n(\omega \ll 1 / \beta) \approx 1 / \beta \omega$ since $K_{a \geqslant 0}(\eta \gg 1)$ $\sim \sqrt{\pi / 2 \eta} e^{-\eta}$ implies that the integral has its main contribution for $0<\eta<10$, and in this range $\omega \ll 1 / \beta$. Hence, after some algebra we obtain

$$
\frac{d \mathcal{P}^{\mathrm{em}}\left(\theta_{0}\right)}{d \Omega}=\frac{d \mathcal{P}_{\mathrm{vac}}^{\mathrm{em}}\left(\theta_{0}\right)}{d \Omega}+\frac{d \mathcal{P}_{\text {ther }}^{\mathrm{em}}\left(\theta_{0}\right)}{d \Omega},
$$

where

where 


$$
\begin{aligned}
\left.\frac{d \mathcal{P}_{\text {ther }}^{\mathrm{em}}\left(\theta_{0}\right)}{d \Omega}\right|_{|t|<50}= & \frac{\Gamma^{2}(2 / 3) \Gamma(8 / 3) \zeta(8 / 3)}{5 \times 48^{1 / 6} \pi^{3}} \frac{\gamma^{-7}}{\tau_{0}}\left(\frac{\beta^{-1}}{\omega_{0}}\right)^{8 / 3}\left\{\frac{\Gamma^{2}(1 / 3) \Gamma(10 / 3) \zeta(10 / 3)}{6^{2 / 3} \Gamma^{2}(2 / 3) \Gamma(8 / 3) \zeta(8 / 3)} \frac{\sin ^{2} \theta_{0}}{\gamma^{2}}\left(\frac{\beta^{-1}}{\omega_{0}}\right)^{2 / 3}\right. \\
& +\left(\frac{32}{3}\right)^{1 / 3} \frac{\Gamma(1 / 3) \zeta(3)}{\Gamma(2 / 3) \Gamma(8 / 3) \zeta(8 / 3)} \frac{\cos \theta_{0}}{\gamma}\left(\frac{\beta^{-1}}{\omega_{0}}\right)^{1 / 3} \\
& \left.+\frac{\left(1+\cos ^{2} \theta_{0}\right)}{2}\left[\frac{\Gamma^{2}(1 / 3) \Gamma(10 / 3) \zeta(10 / 3)}{6^{2 / 3} \Gamma^{2}(2 / 3) \Gamma(8 / 3) \zeta(8 / 3)} \frac{\left(1+t^{2}\right)}{\gamma^{2}}\left(\frac{\beta^{-1}}{\omega_{0}}\right)^{2 / 3}+1\right]\right\}, \\
\left.\frac{d \mathcal{P}_{\text {ther }}^{\mathrm{em}}\left(\theta_{0}\right)}{d \Omega}\right|_{|t|>9 \times 10^{2}} & =\frac{\sqrt{3}}{48 \pi} \frac{\left(1+t^{2}\right)^{-7 / 2}}{\tau_{0} \omega_{0} \beta \gamma^{2}}\left\{\sin ^{2} \theta_{0}+\frac{6}{5}\left(1+\cos ^{2} \theta_{0}\right)\left(1+t^{2}\right)+\frac{64}{5 \sqrt{3} \pi} \sqrt{1+t^{2}} \cos \theta_{0}\right\},
\end{aligned}
$$

and

$$
\begin{aligned}
\frac{d \mathcal{P}_{\mathrm{vac}}^{\mathrm{em}}\left(\theta_{0}\right)}{d \Omega}= & \frac{16}{45 \pi^{2}} \frac{\gamma\left(1+t^{2}\right)^{-5}}{\tau_{0}}\left\{\sin ^{2} \theta_{0}+\frac{9}{8}\left(1+\cos ^{2} \theta_{0}\right)\right. \\
& \left.\times\left(1+t^{2}\right)+\frac{105 \sqrt{3} \pi}{256} \sqrt{1+t^{2}} \cos \theta_{0}\right\} .
\end{aligned}
$$

Equations (2.12) and (2.13) are plotted in Fig. 1 over the result obtained through explicit numerical integration, and are in perfect agreement. Figure 2 plots $d \mathcal{P}_{\text {ther }}^{\mathrm{em}}\left(\theta_{0}\right) / d \Omega$ against $d \mathcal{P}_{\text {vac }}^{\text {em }}\left(\theta_{0}\right) / d \Omega$, and shows that for "large" $|\theta \sin \phi|$, the spin-flip photon emission is largely dominated by the presence of the thermal bath. In particular at the LEP for $|t| \approx 10^{5} \quad(\theta=\phi=\pi / 2), \quad$ we have $\left[d \mathcal{P}_{\text {ther }}^{\mathrm{em}}\left(\theta_{0}\right) / d \Omega\right] /$ $\left[d \mathcal{P}_{\mathrm{vac}}^{\mathrm{em}}\left(\theta_{0}\right) / d \Omega\right] \approx 10^{8}$. This shows that the background thermal bath must not be always overlooked here.

The angular distribution of the radiated power is obtained by multiplying Eq. (2.4) by $\omega$ and integrating over frequencies. By using the same approximations described above, we obtain

$$
\frac{d \mathcal{W}^{\mathrm{em}}\left(\theta_{0}\right)}{d \Omega}=\frac{d \mathcal{W}_{\mathrm{vac}}^{\mathrm{em}}\left(\theta_{0}\right)}{d \Omega}+\frac{d \mathcal{W}_{\text {ther }}^{\mathrm{em}}\left(\theta_{0}\right)}{d \Omega},
$$

where (see Figs. 3 and 4)

$$
\begin{aligned}
&\left.\frac{d \mathcal{W}_{\text {ther }}^{\mathrm{em}}\left(\theta_{0}\right)}{d \Omega}\right|_{\mid t<50}= \frac{\sqrt{3}}{45 \pi^{3}} \frac{\omega_{0}}{\tau_{0} \gamma^{7}}\left(\frac{\beta^{-1}}{\omega_{0}}\right)^{11 / 3}\left\{\left(\frac{3}{4}\right)^{2 / 3} \Gamma^{2}(1 / 3) \Gamma(13 / 3) \zeta(13 / 3) \frac{\sin ^{2} \theta_{0}}{\gamma^{2}}\left(\frac{\beta^{-1}}{\omega_{0}}\right)^{2 / 3}+\left(\frac{9}{32}\right)^{1 / 3}\left(1+\cos ^{2} \theta_{0}\right)\right. \\
& \times\left[\frac{\Gamma^{2}(1 / 3) \Gamma(13 / 3) \zeta(13 / 3)}{2^{2 / 3}} \frac{\left(1+t^{2}\right)}{\gamma^{2}}\left(\frac{\beta^{-1}}{\omega_{0}}\right)^{2 / 3}+\frac{\Gamma^{2}(2 / 3) \Gamma(11 / 3) \zeta(11 / 3)}{3^{-2 / 3}}\right] \\
&\left.+\frac{36 \pi \zeta(4)}{\sqrt{3}} \frac{\cos \theta_{0}}{\gamma}\left(\frac{\beta^{-1}}{\omega_{0}}\right)^{1 / 3}\right\}, \\
&\left.\frac{d \mathcal{W}_{\text {ther }}^{\mathrm{em}}\left(\theta_{0}\right)}{d \Omega}\right|_{|t|>9 \times 10^{2}}=\frac{16}{45 \pi^{2}} \frac{\gamma\left(1+t^{2}\right)^{-5}}{\tau_{0} \beta}\left\{\sin ^{2} \theta_{0}+\frac{9}{8}\left(1+\cos ^{2} \theta_{0}\right)\left(1+t^{2}\right)+\frac{105 \sqrt{3} \pi}{256} \sqrt{1+t^{2}} \cos \theta_{0}\right\},
\end{aligned}
$$

and

$$
\begin{aligned}
\frac{d \mathcal{W}_{\mathrm{vac}}^{\mathrm{em}}\left(\theta_{0}\right)}{d \Omega}= & \frac{77 \sqrt{3}}{256 \pi} \frac{\gamma^{4} \omega_{0}}{\left(1+t^{2}\right)^{13 / 2} \tau_{0}}\left\{\sin ^{2} \theta_{0}+\frac{12}{11} \frac{\left(1+\cos ^{2} \theta_{0}\right)}{\left(1+t^{2}\right)}\right. \\
& \left.+\frac{8192 \sqrt{3}}{2079 \pi} \sqrt{1+t^{2}} \cos \theta_{0}\right\} .
\end{aligned}
$$

\section{FREQUENCY DISTRIBUTION}

The frequency distribution of emitted photons can be obtained by integrating Eq. (2.4) in the solid angle. By using the approximation [10] $d \Omega \approx(2 \pi / \gamma) d t$ which is good for small $\theta$, we obtain

$$
\begin{aligned}
\frac{d \mathcal{P}^{\mathrm{em}}\left(\theta_{0}\right)}{d \omega}= & \frac{3}{10 \pi} \frac{\nu^{2}}{\gamma^{3} \omega_{0} \tau_{0}}\left[\sin ^{2} \theta_{0} \int_{\nu}^{\infty} K_{1 / 3}(s) d s\right. \\
& \left.+\left(1+\cos ^{2} \theta_{0}\right) K_{2 / 3}(\nu)+2 \cos \theta_{0} K_{1 / 3}(\nu)\right] \\
& \times[1+n(\omega)] .
\end{aligned}
$$

The small-angle approximation above is corroborated by the 


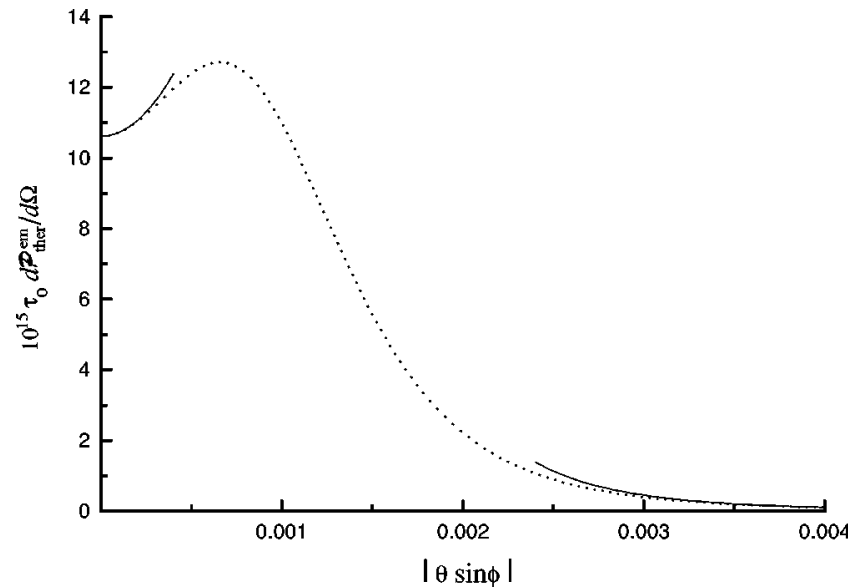

FIG. 1. Thermal contribution to the angular distribution of radiation induced by the deexcitation of electrons at the LEP. The dashed line was obtained through numerical integration and is to be compared with the solid line obtained through analytic approximation. The analogous figure for excitation is very similar.

last section results (see Figs. 1 and 2). The unit in the square brackets is related to the vacuum (see Ref. [10]) and accounts for spontaneous emission, while the $n(\omega)$ term is related to the background thermal bath and accounts for stimulated emission.

The frequency distribution of the radiated power is trivially obtained from this result by simply multiplying Eq. (3.1) by $\omega$, and is introduced for sake of completeness:

$$
\begin{aligned}
\frac{d \mathcal{W}^{\mathrm{em}}\left(\theta_{0}\right)}{d \omega}= & \frac{3}{10 \pi} \frac{\nu^{2} \omega}{\gamma^{3} \omega_{0} \tau_{0}}\left[\sin ^{2} \theta_{0} \int_{\nu}^{\infty} K_{1 / 3}(s) d s\right. \\
& \left.+\left(1+\cos ^{2} \theta_{0}\right) K_{2 / 3}(\nu)+2 \cos \theta_{0} K_{1 / 3}(\nu)\right] \\
& \times[1+n(\omega)] .
\end{aligned}
$$

These results will be used in the next section to calculate the total photon emission and power radiated.

\section{TOTAL EMISSION RATE AND RADIATED POWER}

In order to calculate the total photon emission rate and radiated power, we integrate Eqs. (3.1) and (3.2) in frequencies. The vacuum term is trivially integrated. For LEP parameters and $\gamma>3 \times 10^{3}$, in order to integrate the thermal term, we use the approximation $K_{a>0}(\nu \ll 1)$ $\approx \Gamma(a) 2^{a-1} / \nu^{a}$, since $n(\omega \beta \gg 1) \sim e^{-\omega \beta}$ implies that the integral has its main contribution for $0<\omega \beta<10$ and in this interval $\nu \ll 1$. Now, if $10 \lesssim \gamma \lesssim 10^{2}$, in order to integrate the thermal term we use the approximation $n(\omega \ll 1 / \beta)$ $\approx 1 /(\omega \beta)$ since $K_{a \geqslant 0}(\nu \gg 1) \sim \sqrt{\pi / 2 \nu} e^{-\nu}$ implies that the integral has its main contribution for $0<\nu<10$, and in this interval $\omega \ll 1 / \beta$. In doing these approximations, one must keep in mind that Eq. (2.1) and the assumption in our last section, $d \Omega \approx(2 \pi / \gamma) d t$, are only valid in relativistic regimes. In summary, we obtain, for the total emission rate,

$$
\mathcal{P}^{\mathrm{em}}\left(\theta_{0}\right)=\mathcal{P}_{\text {vac }}^{\mathrm{em}}\left(\theta_{0}\right)+\mathcal{P}_{\text {ther }}^{\mathrm{em}}\left(\theta_{0}\right)
$$

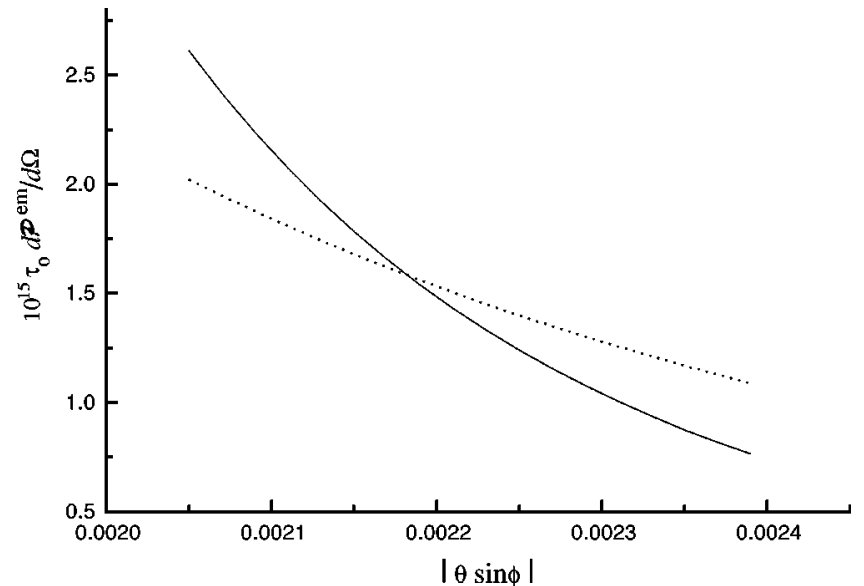

FIG. 2. The dashed line represents $d \mathcal{P}_{\text {ther }}^{\mathrm{em}}\left(\theta_{0}\right) / d \Omega$ while the solid line represents $d \mathcal{P}_{\text {vac }}^{\mathrm{em}}\left(\theta_{0}\right) / d \Omega$. For sufficiently "large", $|\theta \sin \phi|$, the spin-flip photon emission is dominated by the presence of the thermal bath.

where

$$
\begin{aligned}
\mathcal{P}_{\text {ther }}^{\mathrm{em}}\left(\theta_{0}\right)= & \frac{8}{\sqrt{3} \tau_{0}\left(3 \omega_{0} \beta\right)^{8 / 3} \gamma^{8}}\left(\frac{2}{5} \zeta(7 / 3)\left(3 \omega_{0} \beta\right)^{1 / 3} \gamma\right. \\
& \left.+\zeta(8 / 3) \cos \theta_{0}\right)
\end{aligned}
$$

for $\gamma>3 \times 10^{3}$,

$$
\mathcal{P}_{\text {ther }}^{\text {em }}\left(\theta_{0}\right)=\frac{1}{5 \tau_{0} \omega_{0} \beta \gamma^{3}}\left(\frac{2}{\sqrt{3}}+\cos \theta_{0}\right)
$$

for $10 \lesssim \gamma \lesssim 10^{2}$, and

$$
\mathcal{P}_{\mathrm{vac}}^{\mathrm{em}}\left(\theta_{0}\right)=\frac{1}{2 \tau_{0}}\left(1+\frac{8}{5 \sqrt{3}} \cos \theta_{0}\right)
$$

for any $\gamma$, where we assume $\theta_{0}=0$ for deexcitation and $\theta_{0}$ $=\pi$ for excitation because hereafter we will suppose the polarization to be measured along the magnetic field direction. Analogously, we obtain, for the total radiated power,

$$
\mathcal{W}^{\mathrm{em}}\left(\theta_{0}\right)=\mathcal{W}_{\mathrm{vac}}^{\mathrm{em}}\left(\theta_{0}\right)+\mathcal{W}_{\text {ther }}^{\mathrm{em}}\left(\theta_{0}\right)
$$

where

$$
\begin{aligned}
\mathcal{W}_{\text {ther }}^{\text {em }}\left(\theta_{0}\right)= & \frac{16 \omega_{0}}{405 \tau_{0}\left(\omega_{0} \beta\right)^{11 / 3} \gamma^{8}}\left[7 \sqrt{3} \zeta(10 / 3)\left(\omega_{0} \beta\right)^{1 / 3} \gamma\right. \\
& \left.+20 \zeta(11 / 3) \cos \theta_{0}\right]
\end{aligned}
$$

for $\gamma>3 \times 10^{3}$,

$$
\mathcal{W}_{\text {ther }}^{\text {em }}\left(\theta_{0}\right)=\frac{\beta^{-1}}{2 \tau_{0}}\left(1+\frac{8}{5 \sqrt{3}} \cos \theta_{0}\right)
$$

for $10<\gamma<10^{2}$, and 


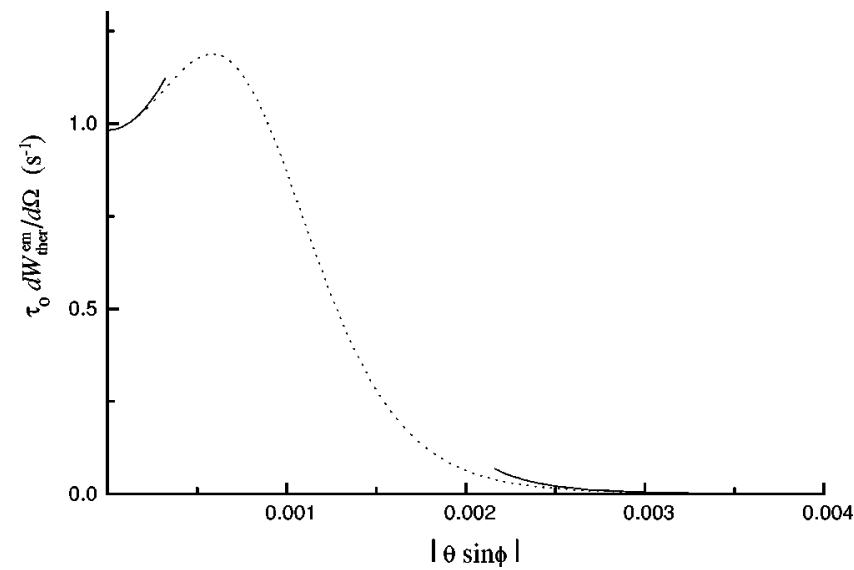

FIG. 3. Thermal contribution to the angular distribution of the radiated power induced by the deexcitation of electrons at the LEP. The dashed line was obtained through numerical integration and is to be compared with the solid curves obtained through analytic approximations. The analogous figure for excitation is very similar.

$$
\mathcal{W}_{\mathrm{vac}}^{\mathrm{em}}\left(\theta_{0}\right)=\frac{16 \omega_{0} \gamma^{3}}{5 \sqrt{3} \tau_{0}}\left(1+\frac{35 \sqrt{3}}{64} \cos \theta_{0}\right)
$$

for any $\gamma$. In particular, for the LEP we have

$$
\begin{aligned}
& \mathcal{P}_{\text {ther }}^{\mathrm{em}}\left(\theta_{0}\right)=\left(4 \times 10^{-16}+6 \times 10^{-18} \cos \theta_{0}\right) / 2 \tau_{0}, \\
& \mathcal{W}_{\text {ther }}^{\mathrm{em}}\left(\theta_{0}\right)=\left(4 \times 10^{-2}+5 \times 10^{-4} \cos \theta_{0}\right) / 2 \tau_{0},
\end{aligned}
$$

which are much smaller than

$$
\begin{gathered}
\mathcal{P}_{\text {vac }}^{\mathrm{em}}\left(\theta_{0}\right)=\left(1+9 \times 10^{-1} \cos \theta_{0}\right) / 2 \tau_{0}, \\
\mathcal{W}_{\mathrm{vac}}^{\mathrm{em}}\left(\theta_{0}\right)=\left(4 \times 10^{20}+4 \times 10^{20} \cos \theta_{0}\right) / 2 \tau_{0},
\end{gathered}
$$

respectively. This result shows that eventually the background thermal-bath contribution to the total transition rate is very small in this case, and can be disregarded for depolarization purposes. This will be explicitly shown in the next section. Note, however, the strong $\gamma$ dependence on $\mathcal{P}_{\text {ther }}^{\mathrm{em}}\left(\theta_{0}\right)$ and $\mathcal{W}_{\text {ther }}^{\mathrm{em}}\left(\theta_{0}\right)$ which makes the thermal contribution larger than the vacuum contribution in the $10<\gamma<10^{2}$ range. As a consequence, the background thermal bath not only is important to the photon-emission rate for large solid angles at LEP-type accelerators as shown in Sec. II, but could be also important for the polarization itself provided $\gamma$ was considerably smaller.

\section{POLARIZATION}

Finally, let us calculate the polarization function

$$
P=\frac{\mathcal{P}_{\downarrow}-\mathcal{P}_{\uparrow}}{\mathcal{P}_{\downarrow}+\mathcal{P}_{\uparrow}}
$$

for electrons at the LEP taking into account the background thermal bath, where the excitation rate is given by

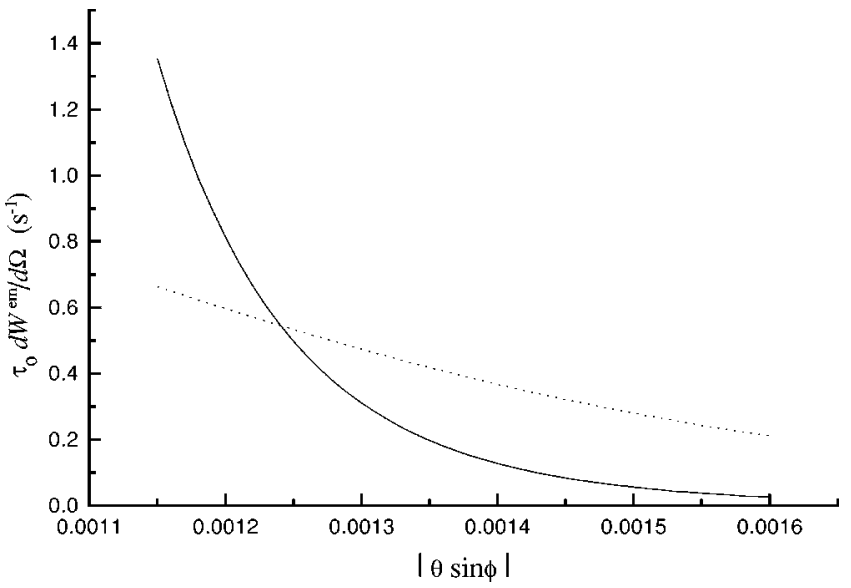

FIG. 4. The dashed line represents $d \mathcal{W}_{\text {ther }}^{\text {em }}\left(\theta_{0}\right) / d \Omega$, while the solid line represents $d \mathcal{W}_{\text {vac }}^{\text {em }}\left(\theta_{0}\right) / d \Omega$. For sufficiently "large", $|\theta \sin \phi|$, the radiated power is dominated by the presence of the thermal bath.

$$
\mathcal{P}_{\uparrow}=\mathcal{P}_{\mathrm{vac}}^{\mathrm{em}}(\pi)+\mathcal{P}_{\text {ther }}^{\mathrm{em}}(\pi)+\mathcal{P}^{\mathrm{abs}}(\pi)
$$

and the deexcitation rate is given by

$$
\mathcal{P}_{\downarrow}=\mathcal{P}_{\text {vac }}^{\text {em }}(0)+\mathcal{P}_{\text {ther }}^{\text {em }}(0)+\mathcal{P}^{\text {abs }}(0)
$$

$\mathcal{P}_{\text {vac }}^{\mathrm{em}}(\pi), \mathcal{P}_{\text {ther }}^{\mathrm{em}}(\pi)$, and $\mathcal{P}^{\text {abs }}(\pi)$ are the excitation rates associated with spontaneous photon emission, stimulated photon emission, and photon absorption, respectively, while $\mathcal{P}_{\text {vac }}^{\mathrm{em}}(0), \mathcal{P}_{\text {ther }}^{\mathrm{em}}(0)$, and $\mathcal{P}^{\mathrm{abs}}(0)$ are the deexcitation rates associated analogously with spontaneous photon emission, stimulated photon emission and photon absorption.

Now, by substituting Eqs. (5.2) and (5.3) into Eq. (5.1), and using Eq. (2.10), we obtain

$$
P \approx P_{\mathrm{vac}}\left(1-2 \frac{\mathcal{P}_{\mathrm{ther}}^{\mathrm{em}}(0)+\mathcal{P}_{\mathrm{ther}}^{\mathrm{em}}(\pi)}{\mathcal{P}_{\mathrm{vac}}^{\mathrm{em}}(0)+\mathcal{P}_{\mathrm{vac}}^{\mathrm{em}}(\pi)}\right),
$$

where $P_{\text {vac }}=0.92$ is the vacuum polarization obtained at zero temperature. Finally, by using Eqs. (4.9) and (4.10), we obtain

$$
P \approx P_{\mathrm{vac}}\left(1-8 \times 10^{-16}\right),
$$

which confirms the statement in the last section that the background thermal bath contribution to the depolarization should be small.

\section{DISCUSSION}

We have discussed the influence of the background thermal bath on the depolarization of electrons in high-energy storage rings, and the corresponding photon emission and radiated power. We have calculated the angle and frequency distribution of such photons and obtained that in a large interval of solid angles the photon emission is enhanced by several orders of magnitude because of the thermal bath. In addition, we have shown that the background thermal bath can be very important to the total photon emission and overall depolarization in some $\gamma$ interval, although it can be ne- 
glected at the LEP and similar accelerators. In spite of the fact that some of these conclusions were anticipated before [12] by modeling the electron's spin flip by the transition of a two-level scalar system, this approximate approach and the realistic calculation here developed lead to fairly different numerical results. This is another indication of the outstanding role played by the Thomas precession in this context as first called attention to by Bell and Leinaas [13] and further investigated in more detail by Barber et al. [14].

\section{ACKNOWLEDGMENTS}

We are really grateful to Desmond Barber for reading carefully our manuscript and for his enlightening comments. We also thank him for calling our attention to Ref. [15]. A.G. and D.V. acknowledge full support by Fundação de Amparo à Pesquisa do Estado de São Paulo, while G.M. was partially supported by Conselho Nacional de Desenvolvimento Científico e Tecnológico.
[1] V. N. Baier, Usp. Fiz. Nauk. 105, 441 (1971) [Sov. Phys. Usp. 14, 695 (1972)]; D. Potaux, in Proceedings of the 8th International Conference on High Energy Accelerators, edited by M. H. Blewett (CERN, Geneve, 1971).

[2] J. G. Learned, L. K. Resvanis, and C. M. Spencer, Phys. Rev. Lett. 35, 1688 (1975).

[3] J. R. Johnson et al., Nucl. Instrum. Methods Phys. Res. 204, 261 (1983).

[4] L. Knudsen et al., Phys. Lett. B 270, 97 (1991).

[5] R. Assmann et al., in High Energy Spin Physics, edited by K. J. Heller and S. L. Smith, AIP Conf. Proc. No. 343 (AIP, New York, 1995).

[6] D. P. Barber et al., Phys. Lett. B 343, 436 (1995).

[7] P. Chiappeta et al., Nucl. Phys. B262, 495 (1985).

[8] A. A. Sokolov and I. M. Ternov, Dokl. Akad. Nauk. SSSR 153, 1052 (1963) [Sov. Phys. Dokl. 8, 1203 (1964)].
[9] Ya. S. Derbenev and A. M. Kondratenko, Zh. Eksp. Teor. Fiz. 64, 1918 (1973) [Sov. Phys. JETP 37, 968 (1973)].

[10] J. D. Jackson, Rev. Mod. Phys. 48, 417 (1976).

[11] D. Treille, in Polarization at LEP, edited by G. Alexander et al. (CERN Yellow report No. 88-06, Geneva, Switzerland, 1988), Vol. 1.

[12] S. S. Costa and G. E. A. Matsas, Phys. Rev. D 52, 3466 (1995).

[13] J. S. Bell and J. M. Leinaas, Nucl. Phys. B212, 131 (1983).

[14] D. P. Barber and S. R. Mane, Phys. Rev. A 37, 456 (1988); D. P. Barber, K. Heinemann, and G. Ripken, Z. Phys. C 64, 117 (1994); 64, 143 (1994).

[15] K. Heinemann, physics/9611001, DESY Report No. 96-226.

[16] P. V. Landshoff and J. C. Taylor, Nucl. Phys. B430, 683 (1994). 